\title{
Correction to: A multiplexed genotyping assay to determine vegetative incompatibility and mating type in Cryphonectria parasitica
}

\author{
C. Cornejo • B. Šever • Q. Kupper • S. Prospero • D. Rigling
}

Published online: 22 June 2019

(C) Koninklijke Nederlandse Planteziektenkundige Vereniging 2019

\section{Correction to: Eur J Plant Pathol \\ https://doi.org/10.1007/s10658-019-01751-w}

Vendor overlooked Fig. $2 \& 3$ during proof correction and need to be replaced with these.

Publisher's note Springer Nature remains neutral with regard to jurisdictional claims in published maps and institutional affiliations.

The online version of the original article can be found at https://doi.org/10.1007/s10658-019-01751-w

C. Cornejo $(\bowtie) \cdot$ B. Šever $\cdot$ Q. Kupper $\cdot$ S. Prospero $\cdot$ D. Rigling

Swiss Federal Research Institute WSL, Zuercherstr. 111, 8903 Birmensdorf, Switzerland e-mail: carolina.cornejo@wsl.ch

B. Šever Institute of Chemistry and Biotechnology, ZHAW, Gruentalstrasse 14, 8820, Waedenswil, Switzerland 


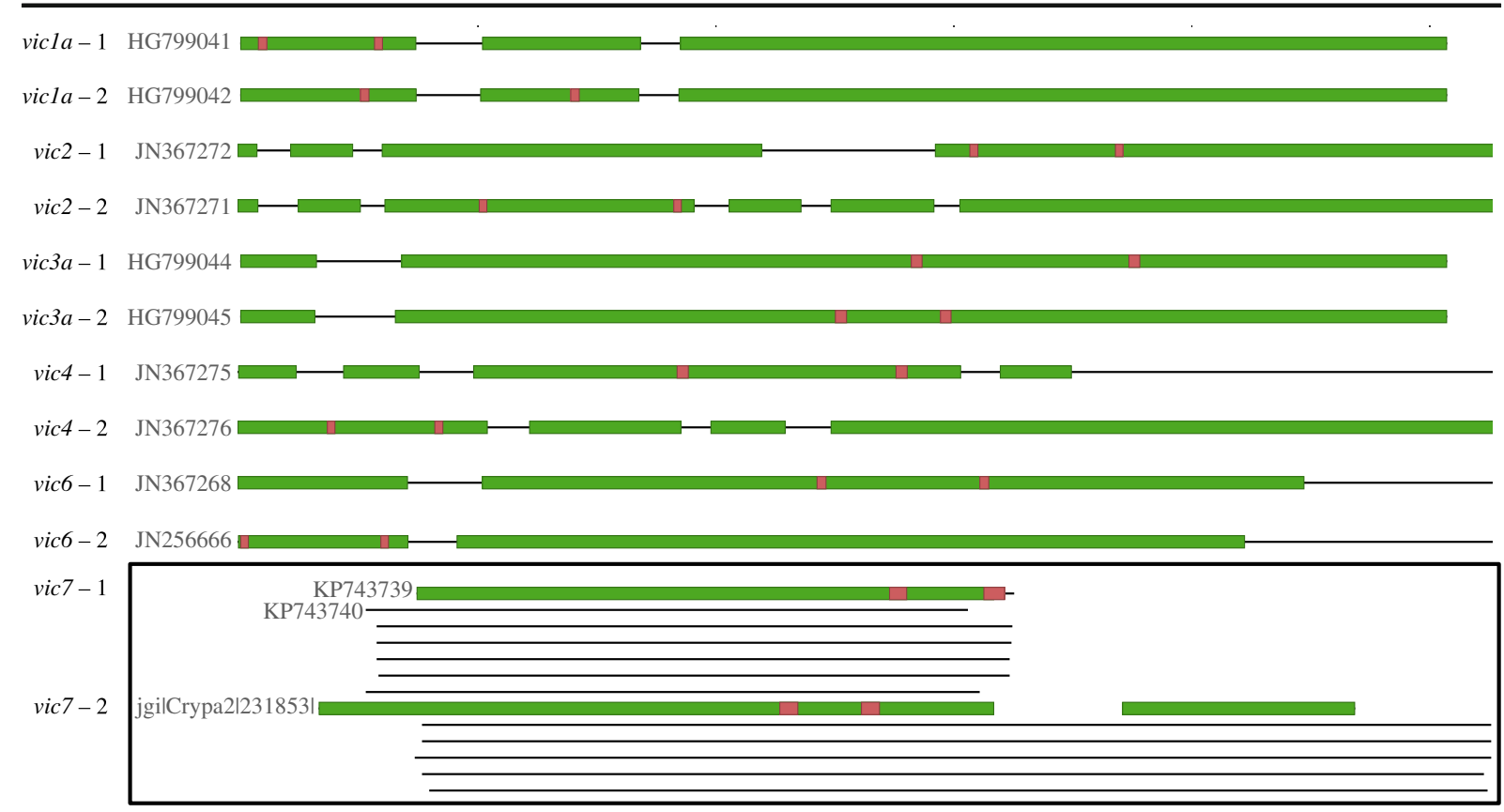

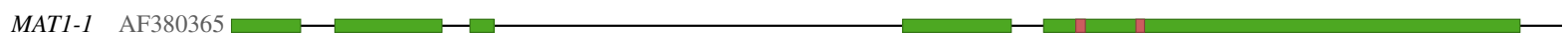

MAT1-2 AF380364

Fig. 2 Map of partial sequences of six di-allelic vic genes and two MAT idiomorphs of Cryphonectria parasitica with primer binding positions. Sequences are designated with their GenBank accession numbers. Black lines represent introns, while green bars denote exon regions and red bars primer sequences. Black box: Alignment of vic7 sequences. For the vic7 locus, ten sequences were generated newly (MH836570 - MH836579; black lines) and analyzed together with KP743739, KP743740, and jgi|Crypa2|231853| to determine the exon and intron regions in the alignment (green bars) 
a

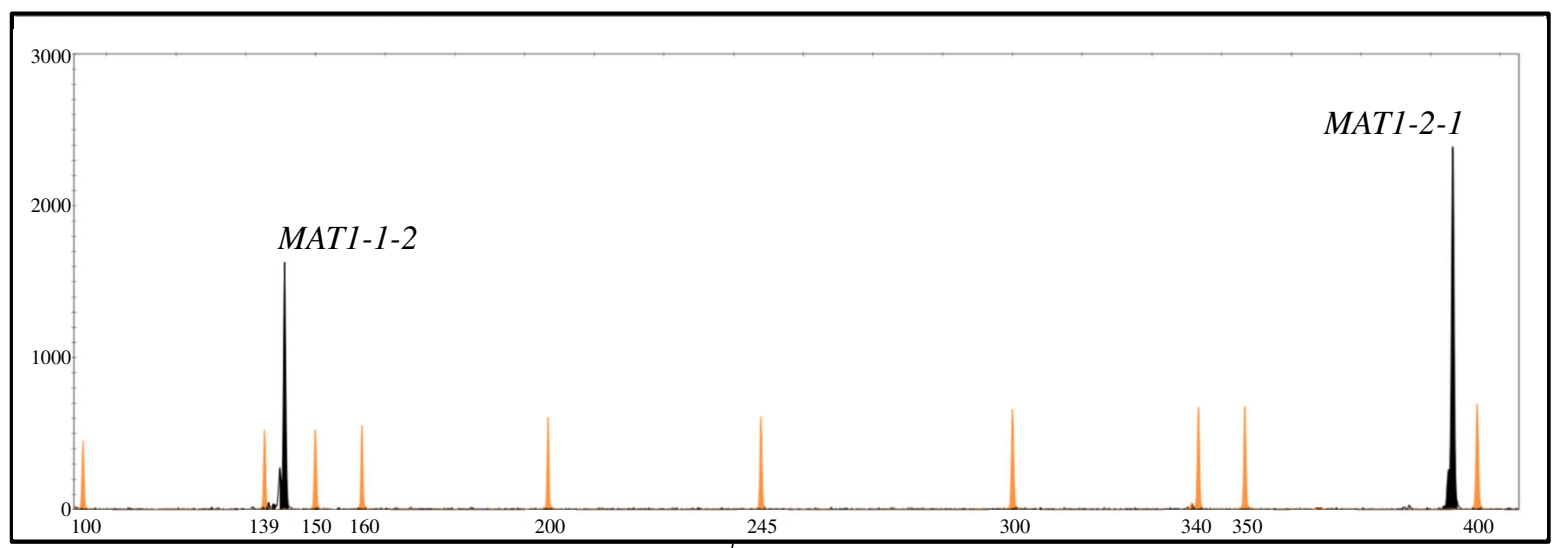

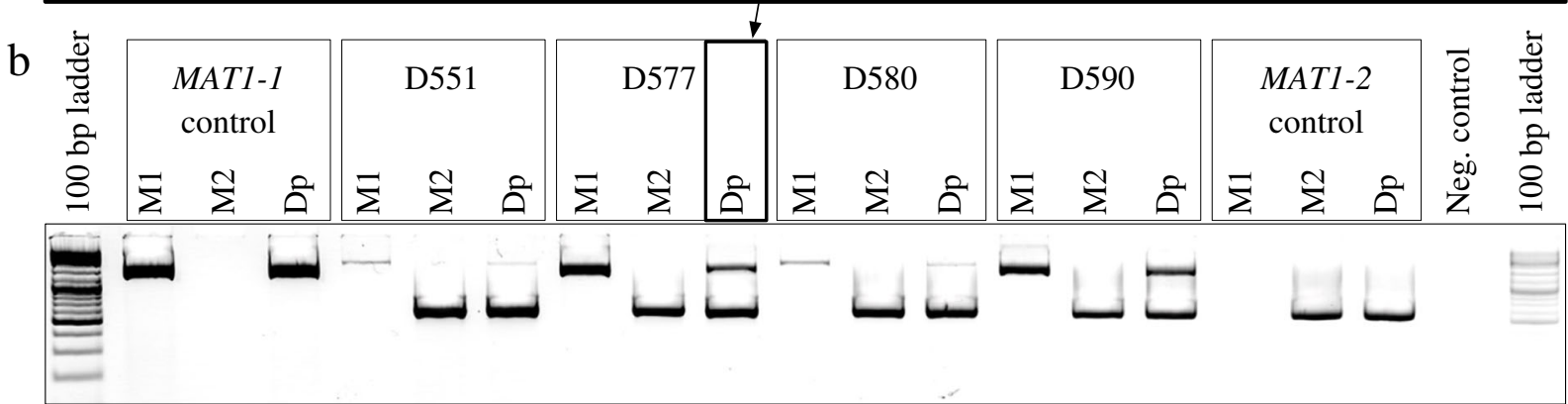

Fig. 3 Detection of both mating types in the same isolate. a Two NED-labeled peaks (black) were present in the same electropherogram of the isolate D577 of Cryphonectria parasitica (orange peaks: DNA size standard). b Verification of heterokaryotic strains applying two single PCR reactions according to Marra and
Milgroom (2001), which amplify the MAT1-1 (M1) and MAT1-2 (M2) loci specifically, and using the same protocol in a duplex (Dp). The concentration of the PCR product yielded varied considerable and the larger fragment (MAT1-1) from the duplex reaction was in a few cases practically invisible on the gel image 